\title{
Étude de cohorte sur la prévalence et les facteurs de risque de complications pulmonaires tardives chez les adultes à la suite d'un traumatisme thoracique mineur fermé
}

\author{
Miville Plourde, MD, MSc*; Marcel Émond, MD, $\mathrm{MSc}^{\dagger}$; André Lavoie, $\mathrm{PhD}^{\dagger}$; \\ Chantal Guimont, MD, PhD ${ }^{\ddagger}$; Natalie Le Sage, MD, MSc ${ }^{\dagger}$; Jean-Marc Chauny, MD, MSc ${ }^{\S}$; \\ Éric Bergeron, $\mathrm{MD}, \mathrm{MSc}^{\dagger}$; Laurent Vanier, $\mathrm{MD}, \mathrm{PhD}^{\|}$; Lynne Moore, $\mathrm{PhD}^{\dagger}$; Nadine Allain- \\ Boulé, MSc, $\mathrm{MBA}^{\dagger}$; Ramona-Florina Fratu, MSc, $\mathrm{BSc}^{\dagger}$; Maryline Dufresne, $\mathrm{BSc}^{\dagger}$
}

\section{RÉSUMÉ}

Objectifs: Les objectifs de cette étude consistent à déterminer la prévalence, les facteurs de risque, et le délai d'apparition de l'hémothorax et du pneumothorax tardifs chez les adultes ayant subi un traumatisme thoracique mineur fermé.

Méthode: Une cohorte prospective de 450 patients consécutifs a été recrutée. Les patients éligibles devaient être âgés de plus de 16 ans, avoir consulté en deçà de 72 heures pour un traumatisme et être disponibles pour un suivi ambulatoire à 2,7 , et 14 jours suivant le traumatisme. Le résultat clinique recherché était la présence d'hémothorax et de pneumothorax tardifs à la radiographie pulmonaire de contrôle.

Résultats: L'hémothorax tardif est survenu chez 11,8\% (IC $95 \%$

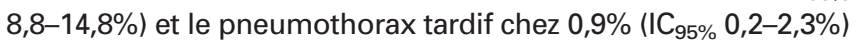
des participants. Au cours de la période de suivi de 14 jours, $87,0 \%$ de ces complications tardives se sont manifestées durant la première semaine. En analyse multivariée, le seul facteur de risque de complications tardives statistiquement significatif était la localisation des fractures sur la radiographie de I'hémithorax. Le rapport de cote ajusté se situe à $1,52\left(\mathrm{IC}_{95 \%}, 0,62-3,73\right)$ pour les côtes inférieures (10-12e côte), à 3,11 (IC $95 \%$ 1,60-6,08) pour les côtes médianes (6-9e côte), et à 5,05 (IC $\mathrm{C}_{95 \%}$ 1,80-14,19) pour les côtes supérieures (troisième-cinquième côte) par rapport aux patients sans fracture.

Conclusion: La présence d'au moins une fracture de côte entre la troisième et la neuvième côte à la radiographie de l'hémithorax est un facteur de risque significatif d'hémothorax et de pneumothorax tardifs.
Keywords: hémothorax retardé, hémothorax tardif, pneumothorax retardé, pneumothorax tardif, traumatisme thoracique

Les traumatismes thoraciques représentent 10 à $15 \%$ des traumatismes rencontrés dans les départements d'urgence. ${ }^{1}$ Plus de $75 \%$ des patients avec fractures de côtes ne requièrent pas d'hospitalisation et sont traités en ambulatoire. ${ }^{2}$ Pourtant, ces derniers sont à risque de développer des complications pulmonaires tardives. ${ }^{3-9}$ La seule étude de cohorte prospective sur le sujet révèle qu'au cours des 2 premières semaines suivant un traumatisme thoracique mineur fermé, $7,4 \%$ des patients avaient développé un hémothorax tardif, et $2,0 \%$ un pneumothorax tardif. Cette étude qui portait sur une population à faible risque a permis d'identifier une corrélation entre le nombre de fractures de côtes et la présence d'hémothorax tardif. ${ }^{3}$ Quant aux études rétrospectives, elles rapportent que le nombre de fractures de côtes, l'âge, et la présence d'emphysème sous-cutané constituent les principaux facteurs de risque de complications pulmonaires tardives. ${ }^{49}$

À l'heure actuelle, aucune étude prospective n'a confirmé ces résultats. Il n'y a donc pas de consensus dans la littérature médicale quant au suivi des patients souffrant d'un traumatisme thoracique mineur fermé. ${ }^{3,8-10}$ Les buts de notre étude prospective sont

Tiré de *Centre hospitalier universitaire de Québec, Hôpital Saint-François d'Assise, Québec, QC; †Axe Traumatologie-Urgence-Soins intensifs, Centre de recherche FRSO du CHA, Québec, QC; $¥$ Axe Reproduction, Santé périnatale et santé de I'enfant, Québec, QC; §Centre de recherche de I'hôpital du Sacré-Cœur de Montréal, Montréal, QC; IIHôpital Charles LeMoyne, Greenfield Park, OC.

Correspondance à: Dr Miville Plourde, Université Laval, Québec, CHUQ - Hôpital Saint-François d'Assise, 10, rue de I'Espinay, QC G1L 3L5; miville. plourde.1@ulaval.ca.

This article has not been peer reviewed. 
d'évaluer la prévalence de l'hémothorax et du pneumothorax tardifs, le moment de leur survenue, et leurs facteurs de risque.

\section{MÉTHODE}

\section{Devis et population}

Les patients de cette cohorte prospective ont été recrutés de façon consécutive du 20 novembre 2006 au 31 décembre 2008 à l'urgence de deux centres universitaires de la région de Québec: l'hôpital de l'Enfant-Jésus, centre de niveau 1 en traumatologie, et le Centre Hospitalier de l'Université Laval, centre non désigné en traumatologie. Les comités d'éthique de ces deux hôpitaux ont approuvé notre protocole de recherche. La rédaction et la présentation des résultats de cet article sont inspirées du « Strobe Statement ${ }^{11}$

\section{Recueil de données}

Les patients éligibles étaient identifiés par un urgentologue à la visite initiale à l'urgence suite à un traumatisme fermé du thorax. Ils devaient présenter au moins l'un des quatre facteurs suivants: 1) une douleur principalement localisée au niveau de la cage thoracique; 2) une abrasion, une contusion, ou une ecchymose au niveau du thorax; 3 ) une fracture de côte suspectée sans validation radiologique; ou 4) une fracture de côte confirmée à la radiographie pulmonaire ou de l'hémithorax. De plus, ceux-ci devaient être âgés d'au moins 16 ans, aptes à consentir à l'étude et disponibles pour trois visites de contrôle à 2,7 , et 14 jours suivant le traumatisme. Étaient exclus les patients qui présentaient l'un des facteurs suivant: 1) une lésion d'origine non traumatique; 2) un traumatisme thoracique survenu plus de 72 heures avant la visite à l'urgence; 3) un traumatisme nécessitant une hospitalisation; 4) une fracture de la première ou de la deuxième côte; 5) une fracture du sternum; 6) une lésion médiastinale; 7) une lésion pulmonaire initiale autre que l'atélectasie (hémothorax, pneumothorax, contusion pulmonaire, pneumonie); ou 8) toute autre blessure non thoracique pour laquelle un traitement ou un suivi orthopédique ou chirurgical était nécessaire. Ces critères visaient à retenir les traumatismes isolés du thorax sans complication aiguë diagnostiquée initialement.

Lors de la visite initiale, l'urgentologue a déterminé l'admissibilité de chaque patient et a obtenu son consentement. Le médecin a complété un questionnaire structuré qui permettait de colliger: 1) les caractéristiques sociodémographiques (âge, sexe); 2) les habitudes de vie (tabagisme, consommation d'alcool); 3) les comorbidités (maladie pulmonaire obstructive chronique, ostéoporose, asthme, diabète); 4) la prise de médicaments (acide acétylsalicylique, clopidogrel, warfarine); 5) le mode d'arrivée à l'urgence (ambulant ou ambulance); 6) le mécanisme du traumatisme; et 7) les symptômes (douleur thoracique, douleur pleurétique, dyspnée à l'effort, dyspnée au repos, hémoptysie, syncope). L'urgentologue a effectué un examen physique standardisé afin de recueillir: 1) les signes vitaux obtenus au triage; 2) les abrasions thoraciques; 3) les contusions ou ecchymoses thoraciques; 4) les crépitements osseux; et 5) l'emphysème sous-cutané. Le patient a également subi des radiographies de l'hémithorax impliqué et des films pulmonaires postéro-antérieurs et latéraux. Les résultats des films ont été interprétés par un radiologiste, à l'aveugle du résultat clinique. Par la suite, le patient a été revu de façon systématique par l'équipe de recherche composée d'une infirmière de recherche et d'un urgentologue à 2 , 7 , et 14 jours suivant le traumatisme. L'ensemble des informations colligées par le médecin de l'urgence était revérifié par l'infirmière de recherche à la première visite de suivi, à l'aveugle du résultat clinique. En cas de désaccord entre l'infirmière de recherche et l'urgentologue, l'infirmière reconfirmait l'information auprès du patient et c'était cette information qui était considérée valide. Ces dates de suivi ont été ajustées à plus ou moins 2 journées d'intervalle afin de tenir compte des fins de semaines et des congés fériés. À chaque visite de suivi, le patient a subi une radiographie pulmonaire postéro-antérieure et latérale de contrôle, laquelle a été interprétée par un radiologiste. Les complications tardives recherchées étaient l'hémothorax et le pneumothorax qui n'étaient pas présentes sur la radiographie à la visite initiale, mais qui sont apparues sur les radiographies de contrôle selon le rapport du radiologiste. Plus précisément, nous avons considéré que tout épanchement pleural de novo sur l'une des radiographies pulmonaires de contrôle était attribuable à un hémothorax tardif. Les soins des patients lors de la visite initiale et des visites de suivi à l'urgence ont été laissés à la discrétion du médecin. L'infirmière de recherche a consulté quotidiennement le registre des visites à l'urgence dans le but d'identifier les patients 
manqués. Plusieurs procédures ont été implantées afin de minimiser les perdus de vue et les patients manqués. Premièrement, une indemnité financière a été octroyée afin de compenser partiellement les désagréments reliés aux déplacements. Deuxièmement, un rappel téléphonique a été effectué la veille du rendez-vous par l'infirmière de recherche afin de maximiser la participation des patients aux suivis. Troisièmement un protocole de suivi systématique a été implanté pour minimiser le temps d'attente des participants lors de leur visite. Finalement, un rappel mensuel a été effectué auprès des médecins en formation afin diminuer le nombre de patients manqués.

\section{Analyses statistiques}

Des statistiques descriptives ont été utilisées pour décrire les données. Les variables continues ont été regroupées selon les seuils identifiés lors de la recension des écrits pour l'âge (45 et 65 ans) et le nombre de fractures de côtes (deux et trois fractures de côtes).,3,12-16 Quant aux signes vitaux, les valeurs cliniquement significatives suivantes ont été utilisées: 1) fréquence respiratoire $\geq 20 / \mathrm{min}, 2$ ) saturation $\leq$ $93 \%$, 3) fréquence cardiaque $\geq 100 / \mathrm{min}$, 4) tension artérielle systolique $\leq 120 \mathrm{~mm} \mathrm{Hg}$, et 5) tension artérielle diastolique $\leq 80 \mathrm{~mm} \mathrm{Hg}$.

Dans un premier temps, une régression logistique univariée a permis d'isoler l'ensemble des facteurs de risque potentiellement significatifs associés aux complications pulmonaires tardives $(\alpha \leq 0,15) \cdot{ }^{17}$ Dans un second temps, une régression logistique multivariée a été réalisée afin d'identifier les facteurs de risque significatifs associés aux complications pulmonaires tardives $(\alpha \leq 0,05)$. Une méthode de régression logistique descendante manuelle a été préconisée pour isoler les variables statistiquement significatives. ${ }^{18}$ De plus, les facteurs de risque reconnus soit l'âge et le nombre de fractures de côtes ont été conservés dans les modèles. L'évaluation des modèles s'est faite par la statistique $\mathrm{C}$ pour mesurer la discrimination et par le test de Hosmer et Lemeshow pour mesurer la calibration. ${ }^{19}$ L'analyse des données a été effectuée avec le logiciel $S A S$ version 9.2 (SAS Institute, Cary, $\mathrm{NC}$ ).

Selon les données de l'étude de Misthos et collègues, $57 \%$ des patients ont présenté au moins une fracture de côte. Au total, $12.8 \%$ des cas avec au moins une fracture de côte ont eu un diagnostic d'hémothorax retardé comparativement à $0 \%$ chez ceux qui n'avaient pas de fracture de côte. ${ }^{3} \mathrm{Si}$ l'on considère de façon conservatrice que $3 \%$ des patients avec au moins une fracture de côte développeront un diagnostic d'hémothorax retardé il nous faudrait recruter au minimum 210 patients pour obtenir une différence statistiquement significative avec une puissance de $80 \%$ et un $\alpha$ à $5 \%$.

\section{RÉSULTATS}

Des 1,186 patients évalués pour un traumatisme mineur fermé à l'urgence, 556 ont été exclus et 101 ont refusé de participer à l'étude laissant ainsi 529 patients éligibles. Parmi ces patients éligibles, 50 n'ont pas été recrutés lors de leur visite initiale à l'urgence (patients manqués), et 29 ont omis de se présenter à l'ensemble de leurs visites de suivi (patients perdus lors du suivi) pour une population à l'étude de 450 patients (diagramme 1).

Les patients recrutés étaient en moyenne plus âgés que les patients manqués et que les patients perdus lors du suivi (52,5 ans comparativement à 45,7 ans et 42,7 ans, respectivement). Ces premiers présentaient également davantage d'atélectasie au niveau de la radiographique pulmonaire initiale (10,2\% comparativement à $0 \%$ et $3,4 \%)$ et avaient plus de fractures de côtes au niveau de la radiographie de l'hémithorax $(0,66$ fracture comparativement à 0,15 fracture et 0,53 fracture). De plus, ces fractures de côtes étaient davantage localisées au niveau des côtes supérieures (troisième-cinquième côte: $7,1 \%$ comparativement à $0 \%$ et $0 \%$ ) et des côtes médianes (sixième-neuvième côte: $24,4 \%$ comparativement à $8,0 \%$ et $18,2 \%$ ) (tableau 1 ).

Tous les patients recrutés ont eu au moins un suivi aux jours 2, 7, ou 14 post-trauma après l'évaluation initiale à l'urgence (100,0\%). Durant la période de suivi, 288 patients retenus se sont présentés au jour 2 $(64,0 \%), 366$ au jour $7(81,3 \%), 344$ au jour 14 $(76,4 \%)$, et 409 se sont présentés au jour 7 ou au jour 14 (90,9\%). Au total, 12,0\% ( $\mathrm{IC}_{95 \%}$ 9,0-15,0\%) des patients ont présenté au moins une complication tardive durant les premiers 14 jours de suivi: l'hémothorax est apparu chez 53 patients $(11,8 \%$; $\left.\mathrm{IC}_{95 \%} 8,8-14,8 \%\right)$ et le pneumothorax chez quatre patients $\left(0,9 \%\right.$; $\left.\mathrm{IC}_{95 \%} 0,2-2,3 \%\right)$. Plus de la moitié des complications tardives ont été détectées dans les 2 premiers jours $(53,7 \%), 33,3 \%$ entre les jours 2 et 7 , tandis que $13,0 \%$ ont été diagnostiqués durant la 


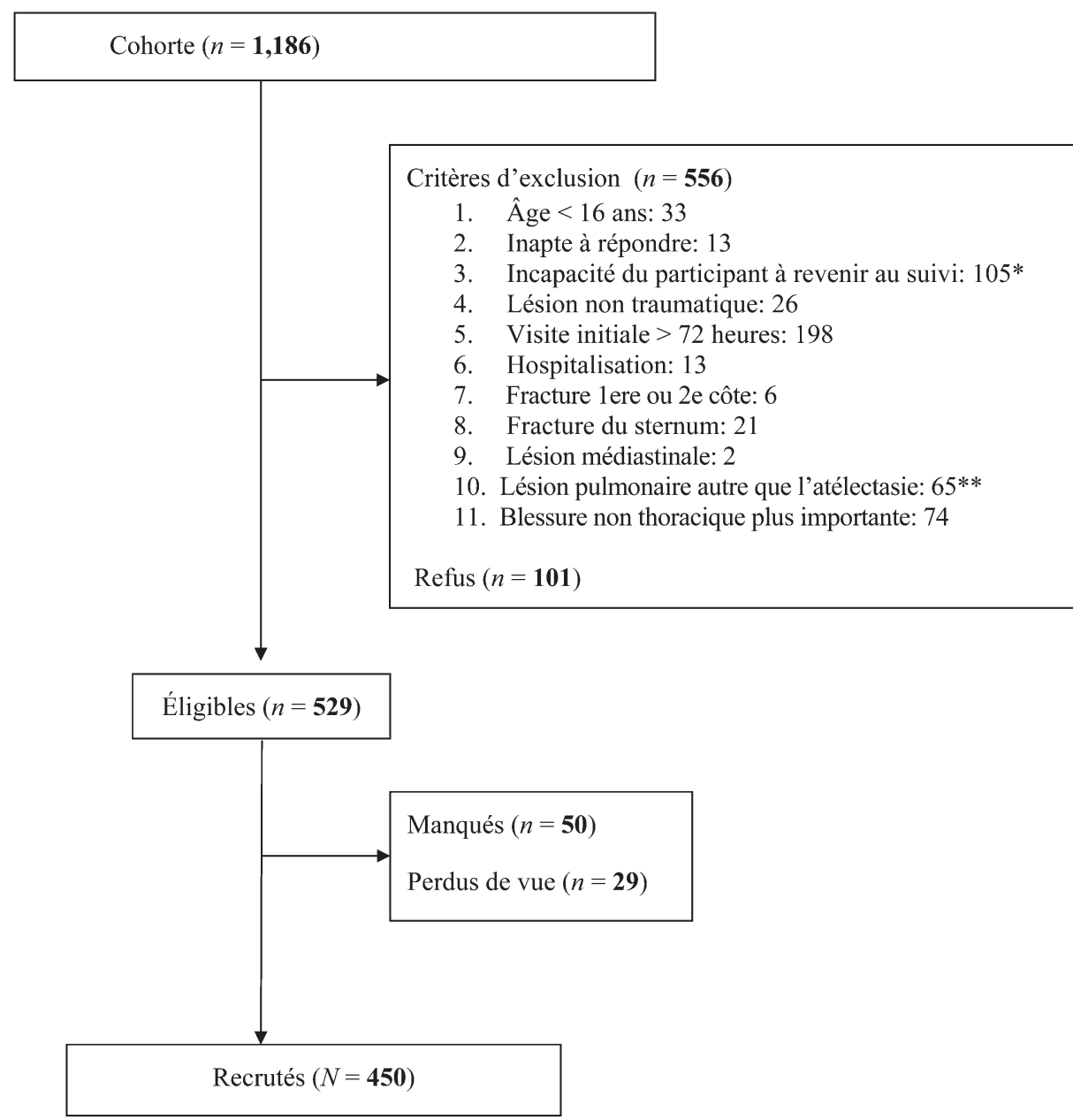

Diagramme 1. Diagramme de flux. *Patients qui n'étaient pas disponibles pour au moins une des visites de suivi. **Hémothorax, pneumothorax, contusion pulmonaire, pneumonie. deuxième semaine post-trauma (tableau 2). Parmi les patients ayant développé une complication retardée, quatre patients ont nécessité une admission d'une durée moyenne de 3,75 jours. Trois de ces derniers ont nécessité un traitement chirurgical.

En analyse univariée, le risque de développer une complication tardive augmente avec l'âge puisque comparé aux patients de moins de 45 ans, le rapport de cote brute se situe à 3,18 (IC $95 \%$ 1,35-7,47) pour les 45 à 64 ans et à 4,08 (IC $95 \%$ 1,64-10,19) pour les plus de 65 ans (tableau 3). Une relation similaire existe par rapport au nombre de fractures de côtes. En effet, comparé aux patients sans fracture de côte, le rapport de cote brute augmente à 3,76

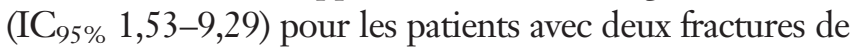
côtes et à 9,51 ( $\left.\mathrm{IC}_{95 \%} 4,03-22,45\right)$ pour les patients avec au moins trois fractures de côtes (tableau 3). Le même type de relation existe à l'égard de la localisation des fractures de côtes puisque le risque augmente à mesure que la hauteur de la fracture de la côte augmente. De fait, le rapport de cote brute comparé à celui des patients qui n'ont pas de fracture de côte passe de 1,53 ( $\mathrm{IC}_{95 \%}$ 0,67-3,48) pour les côtes inférieures (dixième-douzième côte) à 3,22 (IC $95 \%$ 1,72-6,01) pour les côtes médianes (sixième-neuvième côte), et à 4,84 ( $\mathrm{IC}_{95 \%}$ 2,14-10,99) pour les côtes supérieures (troisième-cinquième côte) (tableau 3 ).

Outre l'âge, le nombre de fractures de côtes et la localisation des fractures de côtes, parmi l'ensemble des autres variables étudiées, les variables statistiquement significatives $(\alpha \leq 0,15)$ retenues pour l'élaboration du modèle multivarié ont été les suivantes: l'asthme $(p=0,02)$, le diabète $(p=0,11)$, l'aspirine $(p=0,03)$, l'arrivée en ambulance $(p=0,04)$, un coup direct $(p=0,10)$, la syncope $(p=0,11)$, et l'atélectasie $(p=0,003)$. La régression logistique multivariée révèle que le seul facteur qui demeure statistiquement significatif est la localisation des fractures de côtes. En effet, en gardant les facteurs de risque reconnus à savoir l'âge supérieur à 65 ans et la présence d'au moins trois fractures de côtes, le rapport de cote ajusté passe de $1,52\left(\mathrm{IC}_{95 \%} 0,62-3,73\right)$ pour les côtes inférieures à 
Facteurs de risque de complications pulmonaires tardives suite à un traumatisme thoracique mineur fermé

\begin{tabular}{|c|c|c|c|}
\hline Visite initiale & Recrutés, $n(\%)$ & Manqués, $n(\%)$ & Perdus de vue, $n(\%)$ \\
\hline Nombre de patients & 450 & 50 & 29 \\
\hline Âge $\geq 65$ ans & $103(22,9)$ & $6(12,0)$ & $2(6,9)$ \\
\hline Nombre hommes & $276(61,3)$ & $35(70,0)$ & $15(51,7)$ \\
\hline Arrivé en ambulance & $122(27,1)$ & $11(23,4)$ & $6(20,7)$ \\
\hline \multicolumn{4}{|l|}{ Signes vitaux à l'arrivé } \\
\hline$F R \geq 20 / \min$ & $130(28,9)$ & $18(36,0)$ & $7(24,1)$ \\
\hline Saturation $\leq 93 \%$ AA & $36(8,0)$ & $5(10,0)$ & $4(13,8)$ \\
\hline$F C \geq 100 /$ min & $44(9,8)$ & $8(16,0)$ & $2(6,9)$ \\
\hline $\mathrm{TAS} \leq 120 \mathrm{~mm} \mathrm{Hg}$ & $96(21,3)$ & $11(22,0)$ & $7(24,1)$ \\
\hline $\mathrm{TAD} \leq 80 \mathrm{~mm} \mathrm{Hg}$ & $243(54,0)$ & $30(60,0)$ & $17(58,6)$ \\
\hline \multicolumn{4}{|l|}{ Radiographie pulmonaire } \\
\hline Atélectasie & $46(10,2)$ & $0(0,0)$ & $1(3,4)$ \\
\hline \multicolumn{4}{|c|}{ Radiographie de I'hémithorax } \\
\hline$\geq 1$ fracture & $145(32,2)$ & $4(8,0)$ & $6(20,7)$ \\
\hline$\geq 2$ fracture & $71(15,8)$ & $1(2,0)$ & $1(3,4)$ \\
\hline$\geq 3$ fracture & $31(6,9)$ & $0(0,0)$ & $1(3,4)$ \\
\hline$\geq 1$ fracture supérieure & $32(7,1)$ & $0(0,0)$ & $0(0,0)$ \\
\hline$\geq 1$ fracture médiane & $110(24,4)$ & $4(8,0)$ & $5(17,2)$ \\
\hline$\geq 1$ facture inférieure & $50(11,1)$ & $0(0,0)$ & $2(6,9)$ \\
\hline
\end{tabular}

$3,11\left(\mathrm{IC}_{95 \%}\right.$ 1,60-6,08) pour les côtes médianes et à 5,05 ( $\mathrm{IC}_{95 \%}$ 1,80-14,19) pour les côtes supérieures. Les résultats sont semblables si l'âge de plus de 45 ans et la présence d'au moins deux fractures de côtes sont utilisés dans le modèle multivarié. La présence de complications tardives est donc intimement reliée à la localisation des fractures de côtes. L'âge et le nombre de fractures ne semblent pas constituer des facteurs de risque de complication tardive (tableau 4).

\section{DISCUSSION}

Les forces de cette étude sont nombreuses. Premièrement, à notre connaissance, il s'agit de la plus grande étude prospective nord-américaine de patients consécutifs s'adressant à une population présentant un traumatisme thoracique mineur fermé au département d'urgence.
Deuxièmement, cette étude inclut les patients âgés de plus de 65 ans contrairement à l'étude de Misthos et collègues. ${ }^{3}$ Finalement, la cueillette des facteurs de risques potentiels a été effectuée à l'aveugle de la survenue des complications tardives.

La présente étude a répertorié une prévalence de $12,0 \%$ (IC $95 \%$ 9,0-15,0\%) de complications pulmonaires tardives à 2 semaines suivant un traumatisme thoracique mineur fermé dont 11,8\% (IC $95 \%$ 8,8$14,8 \%$ ) d'hémothorax et $0,9 \%$ ( $\mathrm{IC}_{95 \%} \quad 0,2-2,3 \%$ ) de pneumothorax. Cette prévalence est comparable à celle observée par l'étude de Misthos et collègues qui avait obtenu $7,4 \%$ d'hémothorax et $2,0 \%$ de pneumothorax. ${ }^{3}$ Les résultats de notre étude corroborent les données de la littérature en ce qui concerne le moment d'apparition des complications pulmonaires tardives. En effet, la majorité des complications

Tableau 2. Type et moment d'apparition des complications tardives

\begin{tabular}{lcccc} 
& \multicolumn{3}{c}{ Suivi } \\
\cline { 2 - 3 } Type de complication tardive & 2 jours, $\mathrm{n}(\%)$ & 7 jours, $\mathrm{n}(\%)$ & 14 jours, $\mathrm{n}(\%)$ & Au moins 1 suivi durant 14 jours, $\mathrm{n}(\%)$ \\
\hline Hémothorax & $28(52,8)$ & $18(34,0)$ & $7(13,2)$ & $53(11,8)$ \\
Pneumothorax & $2(50,0)$ & $2(50,0)$ & $0(0)$ & $4(0,9)$ \\
Hémothorax ou & $29(53,7)$ & $18(33,3)$ & $7(13,0)$ & $54(12,0)$ \\
$\quad$ pneumothorax & $288(64,0)$ & $366(81,3)$ & $344(76,4)$ & $450(100,0)$
\end{tabular}




\begin{tabular}{|c|c|c|c|}
\hline Caractéristique & Total, $n(\%)$ & Avec complication, $n(\%)$ & Rapport de cotes brutes (IC à $5 \%$ ) \\
\hline Nombre de patient & 450 & 54 & ND \\
\hline$<45$ ans & $142(31,5)$ & $7(13,0)$ & 1,00 (référence) \\
\hline $45-64$ ans & $205(45,6)$ & $29(53,7)$ & $3,18(1,35-7,47)$ \\
\hline$\geq 65$ ans & $103(22,9)$ & $18(33,3)$ & $4,08(1,64-10,19)$ \\
\hline Homme & $276(61,3)$ & $33(61,1)$ & $0,99(0,55-1,77)$ \\
\hline Arrivé en ambulance & $122(27,1)$ & $31(28,9)$ & $1,86(1,03-3,36)$ \\
\hline \multicolumn{4}{|c|}{ Habitudes et antécédents personnels } \\
\hline Fumeur/ex-fumeur & $204(58,6)$ & $26(57,8)$ & $0,96(0,51-1,81)$ \\
\hline Alcool & $29(6,4)$ & $5(9,3)$ & $1,58(0,58-4,34)$ \\
\hline MPOC & $14(3,2)$ & 0 & $0,37(0,00-2,25)$ \\
\hline Ostéoporose & $32(7,3)$ & $5(9,6)$ & $1,42(0,52-3,85)$ \\
\hline Diabète & $40(9,1)$ & $8(15,1)$ & $1,97(0,86-4,54)$ \\
\hline \multicolumn{4}{|l|}{ Prise de médicaments } \\
\hline Acétylsalicylique & $71(15,8)$ & $14(25,9)$ & $2,08(1,07-4,07)$ \\
\hline Clopidogrel & $6(1,3)$ & 0 & $0,89(0,00-6,30)$ \\
\hline Warfarine & $19(4,2)$ & $4(7,4)$ & $2,03(0,65-6,36)$ \\
\hline \multicolumn{4}{|l|}{ Mécanisme du traumatisme } \\
\hline Chute $\leq$ de sa hauteur & $155(34,4 \%)$ & $20(37,0 \%)$ & $1,14(0,63-2,05)$ \\
\hline Chute $>$ de sa hauteur & $114(25,3)$ & $16(29,6)$ & $1,28(0,68-2,40)$ \\
\hline Véhicule motorisé & $105(23,3)$ & $14(25,9)$ & $1,17(0,61-2,25)$ \\
\hline Piéton versus auto & $2(0,4)$ & 0 & $3,04(0,00-39,30)$ \\
\hline Cycliste versus auto & $3(0,7)$ & 0 & $1,91(0,00-17,92)$ \\
\hline Coup direct & $48(10,7)$ & $2(3,7)$ & $0,29(0,07-1,24)$ \\
\hline Autre mécanisme & $23(5,1)$ & $2(3,7)$ & $0,69(0,16-3,02)$ \\
\hline \multicolumn{4}{|l|}{ Symptômes à l'arrivée } \\
\hline Douleur thoracique & $442(98,2)$ & $54(100,0)$ & 1,52 (0,23-infini) \\
\hline Douleur pleurétique & $227(50,4)$ & $24(44,4)$ & $0,76(0,43-1,35)$ \\
\hline Dyspnée à l'effort & $92(22,4)$ & $12(23,1)$ & $1,04(0,52-2,08)$ \\
\hline Dyspnée au repos & $31(6,9)$ & $4(7,4)$ & $1,09(0,37-3,26)$ \\
\hline Hémoptysie & $6(1,5)$ & 0 & $0,82(0,00-5,81)$ \\
\hline Syncope & $15(3,7)$ & $4(7,7)$ & $2,61(0,80-8,54)$ \\
\hline \multicolumn{4}{|l|}{ Signes à l'arrivée } \\
\hline$F R \geq 20 /$ minute & $130(28,9)$ & $19(35,2)$ & $1,39(0,77-2,54)$ \\
\hline Saturation $\leq 93 \% A A$ & $36(8,0)$ & $4(7,4)$ & $0,91(0,31-2,68)$ \\
\hline$F C \geq 100 /$ minute & $44(9,8)$ & $6(11,1)$ & $1,18(0,47-2,93)$ \\
\hline $\mathrm{TAS} \leq 120 \mathrm{~mm} \mathrm{Hg}$ & $96(21,3)$ & $11(20,4)$ & $0,94(0,46-1,89)$ \\
\hline $\mathrm{TAD} \leq 80 \mathrm{~mm} \mathrm{Hg}$ & $243(54,0)$ & $28(51,9)$ & $0,91(0,51-1,60)$ \\
\hline Abrasion thoracique & $20(4,4)$ & 0 & $0,25(0,00-1,46)$ \\
\hline Contusion thoracique & $62(13,8)$ & $5(9,3)$ & $0,61(0,23-1,59)$ \\
\hline Crépitement osseux & $13(2,9)$ & $1(1,9)$ & $0,60(0,08-4,74)$ \\
\hline \multicolumn{4}{|l|}{ Radiographie pulmonaire } \\
\hline Atélectasie & $46(10,2)$ & $12(22,2)$ & $3,04(1,46-6,32)$ \\
\hline \multicolumn{4}{|c|}{$\begin{array}{l}\text { Radiographie de l'hémithorax: } \\
\text { présence ou absence de fracture de côte }\end{array}$} \\
\hline$\geq 1$ fracture de côte & $145(32,2)$ & $35(64,8)$ & $4,45(2,42-8,21)$ \\
\hline \multicolumn{4}{|c|}{$\begin{array}{l}\text { Radiographie de l'hémithorax: } \\
\text { nombre de fracture(s) de côte }\end{array}$} \\
\hline Aucune fracture & $305(67,8)$ & $19(35,2)$ & 1,00 (référence) \\
\hline 1 fracture de côte & $74(16,4)$ & $15(27,8)$ & $3,83(1,84-7,96)$ \\
\hline 2 fractures de côtes & $40(8,9)$ & $8(14,8)$ & $3,76(1,53-9,29)$ \\
\hline$\geq 3$ fractures de côtes & $31(6,9)$ & $12(22,2)$ & $9,51(4,03-22,45)$ \\
\hline
\end{tabular}


Facteurs de risque de complications pulmonaires tardives suite à un traumatisme thoracique mineur fermé

\begin{tabular}{|c|c|c|c|}
\hline Caractéristique & Total, $n(\%)$ & Avec complication, $n(\%)$ & Rapport de cotes brutes (IC à 5\%) \\
\hline \multicolumn{4}{|l|}{$\begin{array}{l}\text { Radiographie de l'hémithorax: } \\
\text { localisation des fractures de côtes }\end{array}$} \\
\hline Aucune fracture & $305(67,8)$ & $19(35,2)$ & 1,00 (référence) \\
\hline Côtes supérieures (3-4-5 côtes) & $32(7,1)$ & $13(24,1)$ & $4,84(2,14-10,99)$ \\
\hline Côtes médianes (6-7-8-9 côtes) & $110(24,4)$ & $28(51,8)$ & $3,22(1,72-6,01)$ \\
\hline Côtes inférieures (10-11-12 côtes) & $50(11,1)$ & $10(18,5)$ & $1,53(0,67-3,48)$ \\
\hline
\end{tabular}

surviennent moins de 7 jours suivant le traumatisme., ${ }^{3,-9,20}$ De fait, $87,0 \%$ des complications tardives de notre étude ont été diagnostiquées durant la première semaine et seulement $13,0 \%$ l'ont été durant la seconde semaine post-traumatique.

La revue des écrits antérieurs au niveau des facteurs de risque indique que l'âge et le nombre de fractures de côtes sont des facteurs de risque importants de complications tardives. ${ }^{3-9}$ Notre étude prospective jette un regard différent en examinant une nouvelle variable, soit la localisation des fractures qui rend les facteurs précédents secondaires. En effet, après ajustement pour l'ensemble des facteurs potentiellement confondants, le seul facteur de risque qui demeure statistiquement significatif est la localisation des fractures de côtes. Il semble que ce ne soit pas la quantité, mais bien la localisation de ces fractures qui détermine le risque de complication tardive. Notre étude est la première à démontrer l'importance de la localisation des fractures de côtes, telle qu'évaluée à la radiographie de l'hémithorax, à l'égard du risque de

\begin{tabular}{|c|c|c|}
\hline Caractéristique & $\begin{array}{l}\text { Rapport de cote } \\
\text { (IC à 95\%) }\end{array}$ & $p$ value \\
\hline \multicolumn{3}{|l|}{ Âge } \\
\hline$<65$ ans & 1,00 (référence) & \\
\hline$\geq 65$ ans & $1,62(0,84-3,13)$ & 0,15 \\
\hline \multicolumn{3}{|c|}{ Nombre de fractures de côtes } \\
\hline$<3$ & 1,00 (référence) & \\
\hline$\geq 3$ & $0,95(0,29-3,07)$ & 0,93 \\
\hline \multicolumn{3}{|c|}{ Localisation des fractures de côtes } \\
\hline Aucune & 1,00 (référence) & \\
\hline Côtes supérieures & $5,05(1,80-14,19)$ & 0,002 \\
\hline Côtes médianes & $3,11(1,60-6,08)$ & 0,0009 \\
\hline Côtes inférieures & $1,52(0,62-3,73)$ & 0,36 \\
\hline
\end{tabular}

complications pulmonaires tardives. Nos données préliminaires révèlent que les fractures des côtes 3 à 9 sont un facteur de risque de complications pulmonaires tardives. Une autre étude portant sur les complications aiguës plutôt que tardives identifiaient les fractures de la neuvième à la douzième côte comme étant un facteur prédictif de complications intra-abdominales aiguës, mais ne permettait pas de démontrer le lien entre la localisation des fractures de côtes et la présence de complications intra-thoraciques aiguës. ${ }^{21} \mathrm{Il}$ semble donc que la localisation des fractures de côtes joue un rôle au niveau des complications aigues intra-abdominales et des complications pulmonaires tardives.

Malgré ses forces, notre étude présente toutefois certaines limites. Premièrement, au total $14,9 \%$ des patients de la cohorte ont été manqués ou perdus de vue. L'état de ces patients était en moyenne moins sévère que celui des patients analysés. Il est donc peu probable que ces patients manqués ou perdus de vue aient biaisé l'identification des facteurs de risque. Cependant, ce biais de sélection peut avoir entraîné une légère surestimation de la prévalence de complications tardives au sein de cette cohorte compte tenu qu'il s'agit de patients à faible risque. Deuxièmement, $76,4 \%$ des patients se sont présentés pour un suivi au 14 e jour. Il est donc possible que nous ayons manqué des complications tardives. Néanmoins, plus de $90 \%$ de la cohorte a eu au moins un suivi aux jours 7 ou 14, ce qui minimise ce risque compte tenu que la majorité des complications surviennent durant la première semaine suivant le traumatisme thoracique mineur fermé. Troisièmement, nous n'avons pas mesuré d'accord interjuge au niveau de la mesure des variables dépendantes et indépendantes à l'étude. Quatrièmement, il est possible que certains facteurs de risques potentiels rare ont possiblement été exclues du modèle compte tenu de leur faible prévalence au sein de cette cohorte et ce malgré leur possible effet prédictif. Finalement, les 
complications pulmonaires tardives sont définies par un diagnostic radiologique et non clinique. Seulement une minorité des complications chirurgicales ont nécessité un traitement chirurgical ce qui diverge de l'étude de Misthos et collègues où $84,6 \%$ des hémothorax tardifs ont nécessité un traitement chirurgical comparativement à $42,9 \%$ des pneumothorax tardifs. ${ }^{3}$ Notre devis d'étude n'était pas destiné à évaluer la pertinence de drainer ou non ces complications chirurgicales tardives et elles étaient laissées à la discrétion du médecin traitant. Compte tenu de sa prévalence, une prochaine étude devrait se pencher sur la répercussion clinique de

Intérêts concurrentiels: aucun intérêt déclaré

ces anomalies radiologiques.

\section{CONCLUSION}

L'hémothorax tardif est une conséquence fréquente du traumatisme thoracique mineur fermé. Il survient généralement moins de 2 semaines après l'incident. À la radiographie de l'hémithorax, la présence de fractures de côtes entre la troisième et la neuvième côte est un facteur de risque important de complication tardive.

\section{RÉFÉRENCES}

1. Ziegler DW, Agarwal NN. The morbidity and mortality of rib fractures. I Trauma 1994;37:975-9, doi:10.1097/ 00005373-199412000-00018.

2. Cherry DK, Woodwell DA. National Ambulatory Medical Care Survey: 2000 summary. Adv Data 2002;5:1-32.

3. Misthos P, Kakaris S, Sepsas E, et al. A prospective analysis of occult pneumothorax, delayed pneumothorax and delayed hemothorax after minor blunt thoracic trauma. Eur 7 Cardiothorac Surg 2004;25:859-64, doi:10.1016/j.ejcts. 2004.01.044.

4. Holcomb JB, McMullin NR, Kozar RA, et al. Morbidity from rib fractures increases after age 45. 7 Am Coll Surg 2003;196:549-55, doi:10.1016/S1072-7515(02)01894-X.

5. Lu MS, Huang YK, Liu YH, et al. Delayed pneumothorax complicating minor rib fracture after chest trauma. Am $\mathcal{7}$ Emerg Med 2008;26:551-4, doi:10.1016/j.ajem.2007.08.022.
6. McLoughlin R, Mulcahy R, Kent P, et al. Haemothorax after rib fracture - incidence, timing and prediction. Ir $7 \mathrm{Med}$ Sci 1987;156:117-9, doi:10.1007/BF02954634.

7. Ross RM, Cordoba A. Delayed life-threatening hemothorax associated with rib fractures. I Trauma 1986;26:576-8, doi:10.1097/00005373-198606000-00018.

8. Sharma OP, Hagler S, Oswanski MF. Prevalence of delayed hemothorax in blunt thoracic trauma. Am Surg 2005;71:481-6.

9. Simon BJ, Chu Q, Emhoff TA, et al. Delayed hemothorax after blunt thoracic trauma: an uncommon entity with significant morbidity. 7 Trauma 1998;45:673-6, doi:10.1097/ 00005373-199810000-00005.

10. Shields JF, Emond M, Guimont C, et al. Acute minor thoracic injuries: evaluation of practice and follow-up in the emergency department. Can Fam Phys 2010;56:e117-24.

11. Vandenbroucke JP, von Elm E, Altman DG, et al. Strengthening the Reporting of Observational Studies in Epidemiology (STROBE): explanation and elaboration. Epidemiology 2007;18:805-35, doi:10.1097/EDE.0b013e31 $\underline{81577511 .}$.

12. Barnea $Y$, Kashtan H, Skornick Y, et al. Isolated rib fractures in elderly patients: mortality and morbidity. Can 7 Surg 2002;45:43-6.

13. Bulger EM, Arneson MA, Mock CN, et al. Rib fractures in the elderly. 7 Trauma 2000;48:1040-6; discussion 6-7, doi:10.1097/00005373-200006000-00007.

14. Lee RB, Bass SM, Morris JA Jr, et al. Three or more rib fractures as an indicator for transfer to a Level I trauma center: a population-based study. 7 Trauma 1990;30:689-94, doi:10.1097/00005373-199006000-00006.

15. Sirmali M, Turut H, Topcu S, et al. A comprehensive analysis of traumatic rib fractures: morbidity, mortality and management. Eur 7 Cardiothorac Surg 2003;24:133-8, doi:10.1016/S1010-7940(03)00256-2.

16. Testerman GM. Adverse outcomes in younger rib fracture patients. South Med 7 2006;99:335-9, doi:10.1097/01.smj. 0000203815.29757.d3.

17. Harrell FE Jr, Lee KL, Califf RM, et al. Regression modelling strategies for improved prognostic prediction. Stat Med 1984;3:143-52, doi:10.1002/sim.4780030207.

18. Katz MH. Multivariable analysis: a practical guide for clinicians. New York: Cambridge University Press; 2006.

19. Hosmer DW, Lemeshow S. Applied logistic regression. New York: Wiley-Interscience; 2000.

20. Bundy DW, Tilton DM. Delayed hemothorax after blunt trauma without rib fractures. Mil Med 2003;168:501-2.

21. Al-Hassani A, Abdulrahman H, Afifi I, et al. Rib fracture patterns predict thoracic chest wall and abdominal solid organ injury. Am Surg 2010;76:888-91. 